\title{
Integrating Social Determinants of Health to Precision Medicine through Digital Transformation: An Exploratory Roadmap
}

\author{
Ik-Whan G. Kwon ${ }^{1, * \mathbb{C}}$, Sung-Ho Kim ${ }^{2}$ and David Martin ${ }^{3}$ \\ 1 The Center for Supply Chain Excellence, Saint Louis University, Saint Louis, MO 63108, USA \\ 2 Department of Health Administration, Yonsei University, Seoul 03722, Korea; kimsungho@yonsei.ac.kr \\ 3 The Ancell School of Business, Western Connecticut State University, Danbury, CT 06810, USA; \\ martindg@wcsu.edu \\ * Correspondence: kwoni@slu.edu; Tel.: +1-314-560-5487
}

check for

updates

Citation: Kwon, I.-W.G.; Kim, S.-H.; Martin, D. Integrating Social Determinants of Health to Precision Medicine through Digital Transformation: An Exploratory Roadmap. Int. J. Environ. Res. Public Health 2021, 18, 5018. https:// doi.org/10.3390/ijerph18095018

Academic Editors: Chang Won Lee and Dylan P. Cliff

Received: 29 March 2021

Accepted: 4 May 2021

Published: 10 May 2021

Publisher's Note: MDPI stays neutral with regard to jurisdictional claims in published maps and institutional affiliations.

Copyright: (c) 2021 by the authors. Licensee MDPI, Basel, Switzerland. This article is an open access article distributed under the terms and conditions of the Creative Commons Attribution (CC BY) license (https:/ / creativecommons.org/licenses/by/ $4.0 /)$.

\begin{abstract}
The COVID-19 pandemic has altered healthcare delivery platforms from traditional faceto-face formats to online care through digital tools. The healthcare industry saw a rapid adoption of digital collaborative tools to provide care to patients, regardless of where patients or clinicians were located, while mitigating the risk of exposure to the coronavirus. Information technologies now allow healthcare providers to continue a high level of care for their patients through virtual visits, and to collaborate with other providers in the networks. Population health can be improved by social determinants of health and precision medicine working together. However, these two healthenhancing constructs work independently, resulting in suboptimal health results. This paper argues that artificial intelligence can provide clinical-community linkage that enhances overall population health. An exploratory roadmap is proposed.
\end{abstract}

Keywords: social determinants of health; precision medicine; AI; machine learning; digital transformation; population health; clinical-community linkages; digital divide; digital literacy

\section{Introduction}

The COVID-19 pandemic has altered healthcare delivery platforms from the traditional face-to-face format to online care through digital tools. Unlike the Spanish flu of 1918, which became an international epidemic over the course of a year, COVID-19 spread to every inhabitable continent within weeks, outpacing our health system's ability to test, track, and contain people with suspected infection [1]. The pandemic also caused a dramatic shift in emergency use, as capacity is limited to manage COVID-19-related patients who used emergency rooms as their first treatment places. As a result, there was a dramatic decline in the use of emergency rooms for injuries not related to COVID-19 episodes [2]. In response to this unprecedented health crisis, the healthcare industry saw the rapid adoption of video conferencing and other digital collaboration tools to provide care to patients, regardless of where patients or clinicians were located, while mitigating the risk of exposure to the coronavirus. Information technologies allow healthcare providers to continue a high level of care for their patients via virtual visits and to collaborate with other providers in the networks. In addition, the era of value-based healthcare, digital innovation, big data, and clinical decision support systems (CDSSs) have become vital tools for organizations seeking to improve care delivery. CDSS tools have the ability to analyze large volumes of data and suggest the next steps for treatment, flagging potential problems and enhancing care team efficiency [3].

The information explosion via technology adoption and corresponding data collection on patients has re-oriented our approach to managing population health through the social determinants of health framework (a macro approach) on one hand and how to treat 
individual patients through precision medicine on the other (a micro approach). Indeed, artificial intelligence (AI) technology is necessary to achieve the goal of "precision medicine", where medical decisions and treatments are tailored toward patient specifics. Precision medicine presupposes the availability of massive computing power and algorithms that can learn by themselves (machine learning) at an unprecedented rate [4]. An additional driver of AI technology is the sheer volume of healthcare data due to healthcare experiencing an information boom. The rapid expansion of scientific knowledge and the pace of technological development have resulted in an overwhelming sea of data that is difficult to decipher and apply [5].

Although precision medicine and social determinants of health (SDOH) address the same target (i.e., improving population health), each has been viewed as operating independently from the other, making the national goal of improving population health less effective and costlier. The purpose of this paper is to present an exploratory road map on the role of artificial intelligence in healthcare management. In this paper, $\mathrm{AI}$ is considered as an integrator/communicator between these two constructs: social determinants of health and precision medicine in population health.

This paper is organized as follows. A brief literature survey is presented in Section 2, where we discuss clinical-community linkages using AI as a communicator/integrator of the two health improvement constructs. Research methodology is discussed in Section 3. Based on the literature review, an exploratory roadmap in integrating AI into two health constructs (precision medicine and social determinants of health) is presented in Section 4. Discussion and limitations of this study of using AI in population health management are presented in Section 5. Conclusions are outlined in Section 6.

\section{Literature Review}

Widespread adoption of electronic health records (EHRs) through AI has resulted in the collection of massive amounts of clinical data. The growth of wearable medical devices and the predictive power of diagnostic information with multiple patient characteristics has made AI techniques a promising way to analyze these multimodal data sets [6]. However, extracting and analyzing the wealth of information in an accurate, timely, and reliable manner has been a continual challenge for healthcare providers. Accordingly, digital transformation in healthcare is a natural response to this challenge. Reddy [6] lists several reasons for the digital transformation in the healthcare arena: the rise of on-demand healthcare, the importance of big data in healthcare, treating patients by virtual reality, the growth of wearable medical devices, predictive healthcare management, the wonders of artificial intelligence, blockchain protection on personal and confidential patient information, and the promise of better electronic health records (EHRs). Though some electronic health record systems now allow clinicians to document certain social determinants of health in structured fields, these data are often missing or may be recorded in the format of free, unstructured text [7].

For digital health initiatives to more fully impact upon patient care, medical informatics specialists are investing resources in AI and machine-learning-based algorithms [8]. In some cases, machine-learning-based algorithms are allowing investigators to conduct a more in-depth subgroup analysis of previous published studies, the results of which call into question the practical application of the original investigation [8]. The digital transformation in the healthcare arena also opens a new frontier of on-demand healthcare. One in three American adults have gone online to assess a medical condition, $72 \%$ of internet users say they had looked online for health information within the past year, $47 \%$ of internet users search for information about doctors or other health professionals, and $38 \%$ of internet users search for information about hospitals and other medical facilities [9]. The most commonly searched topics are specific diseases or conditions, treatments or procedures, and doctors or other health professionals. Telemedicine, artificial intelligence (AI)-enabled medical devices, and blockchain electronic health records are just a few concrete examples of the digital transformation in healthcare which are completely reshaping how we interact 
with health professionals, how our data is shared among providers, and how decisions are made about our treatment plans and health outcomes.

On the other hand, the World Health Organization's Report on Digital Health Strategy 2020-2025 [10] addresses population health using the digital transformation of health WHO's position appears to be much broader in scope in targeting population health and not just in precision medicine. The literature on $\mathrm{AI}$ in healthcare in medical intervention has been primarily limited to precision medicine [11-13]. Reasons might be that improving precision medicine may achieve population health. However, such a view addresses only a part of population health. Population health is considered more than the sum of health improvements made by precision medicine.

Population health requires investments in health-related areas in order to improve overall community health. Social determinants of health $(\mathrm{SDOH})$ have been used as the main strategic tool to address community health. WHO defines $\mathrm{SDOH}$ as the conditions in which people are born, grow, live, work, and age. These circumstances are shaped by the distribution of power, money, and resources on global, national, and local levels [14]. The nation's health, according to SDOH theory, depends on the conditions more than medical intervention (precision medicine). In fact, research shows that in order to improve population health, factors that impact the nation's health should be addressed in addition to precision medical care. Research seems to indicate that the social determinants of health have a greater impact on a nation's health than medical intervention. For example, the healthcare delivery system is responsible for only a fraction (about one fifth) of what keeps people healthy. Compared to medical services, $\mathrm{SDOH}$ have two times the responsibility for health outcomes [15]. Indeed, abundant research shows that the population health measured by infant mortality and life expectancy depends more on social determinants such as unemployment rates, alcohol, gun-related homicides, and insurance coverage than medical intervention [16]. However, Kim and Kwon's study [16] did not address the role of $\mathrm{AI}$ in population health.

The Accountable Health Communities Model by the Centers for Medicare and Medicaid Services [17] lists a few of the largest drivers of health care costs that fall outside the clinical care environment (precision medicine). According to a CMS report, social and economic determinants, health behaviors, and the physical environment significantly drive utilization and costs. The evidence seems to be clear that addressing health-related social needs through clinical-community linkages can improve health outcomes and delivery costs.

Broad categories of social determinants of health include social factors, health services, individual behavior, and biology and genetics. It is the interrelationship among these factors that determines individual and population health. However, SDOH data is a messy proposition from the very first step of defining the social determinants of health. People define SDOH differently and inconsistently. Patients do not have "social determinants" per se; they have specific health-related social needs that impact their health and day-to-day lives. SDOH data is a complex collection of disparate data points, which causes difficulties when trying to make inferences [18]. Furthermore, social need screening requires information in the most private and potentially stigmatized areas of patients' lives, including poverty, racism, intimate partner violence, etc. [19]. Only $16 \%$ of physicians and $24 \%$ of hospitals currently screen key social risk domains [20]. A lack of relevant and pertinent data/information on a patient's SDOH could be one of the reasons for such a poor use of SDOH information during the assessment of a patient's health status. AI could play clinical-community linkage roles, connecting with and supplementing information in precision medicine to $\mathrm{SDOH}$ information, making the overall health outcomes better and less costly.

However, it is unclear which social determinants of health best and most strongly relate to clinical and community health interventions, as the list of factors in $\mathrm{SDOH}$ is huge and in many instances uncollectible. To target specific communities for social intervention, some researchers developed a Social Deprivation Index (SDI) that specifically quantifies levels of disadvantage across small areas, evaluates their associations with health out- 
comes, and addresses health inequities. This measure of social deprivation, in combination with other indicators, has potential application in identifying areas that need additional health care resources [21]. Maroko et al. [22] saw deprivation as a state of observable and demonstrable disadvantage relative to the local community where an individual belongs. This disadvantage may occur at various levels as, for example, with regard to food, clothing, housing, education, or work. In fact, a person is considered deprived to the extent that they fall below the level attained by much of the population or below what is considered socially acceptable in the community.

The SDI is a complex index. Nevertheless, it will provide information at a local level where precision medicine can be effectively administrated. Maroko et al. [22] lists a working framework where SDI can be operationalized as shown in Table 1.

Table 1. Factors in the Social Deprivation Index.

\begin{tabular}{|c|c|}
\hline Domain & Variables \\
\hline \multirow{3}{*}{ Education } & $\%$ Population aged 25 years or older with less than 9 years of education \\
\hline & $\%$ Population aged 25 years or older with at least a high school diploma \\
\hline & $\%$ Employed population aged 16 years or older in white-collar occupations \\
\hline \multirow{5}{*}{ Income/employment } & Median family income in US dollars \\
\hline & Income disparity \\
\hline & $\%$ Families below federal poverty level \\
\hline & $\%$ Population below $150 \%$ of federal poverty level \\
\hline & $\%$ Civilian labor force population aged 16 years and older who are unemployed \\
\hline \multirow{5}{*}{ Housing } & Median home value in US dollars \\
\hline & Median gross rent in US dollars \\
\hline & Median monthly mortgage in US dollars \\
\hline & $\%$ Owner-occupied housing units \\
\hline & $\%$ Occupied housing units without complete plumbing \\
\hline \multirow{4}{*}{ Household characteristics } & \% Single-parent households with children younger than 18 \\
\hline & $\%$ Households without a motor vehicle \\
\hline & $\%$ Households without a telephone \\
\hline & $\%$ Households with more than 1 person per room \\
\hline
\end{tabular}

Reproduced with permission from Maroko et al., 2016.

We postulate that medical intervention through $\mathrm{SDOH}$ produces better health outcomes (i.e., in a person's health) with less cost if the consumers (patients) live in a community where social determinants are favorable for fostering community health. Yet, the literature appears to indicate that precision medicine and social determinants of health have developed independently from each other, although these two constructs are related and targeting the same objective: improving population health. A unifying communicator across the healthcare spectrum is needed to integrate these two health improvement tools to achieve the desired population health. We consider that AI should play a clinicalcommunity linkage.

\section{Research Methodology}

This paper discusses a literature review as a methodology for conducting this study. Knowledge production within the field of $\mathrm{AI}$ and precision medicine is accelerating at a tremendous speed, while at the same time remaining fragmented. Although AI and machine learning have been used extensively in industrial and supply chain fields, it is relatively new in the healthcare area to deploy $\mathrm{AI}$ and machine learning as a decisionmaking tool. It is only after the COVID-19 pandemic that people in the healthcare field 
started to pay attention to this decision-making tool. This makes it harder to keep up with the state-of-the-art and to be at the forefront of research, as well as to assess the collective evidence in a particular area of business research [23]. This is the one of reasons why the literature review as a research method is more relevant than before.

\section{Developing an Exploratory Model}

Based on a previous SDOH model by Kim and Kwon [16] and a literature review on clinical-community linkage, the role of digital application to health can be mapped as in Figure 1.

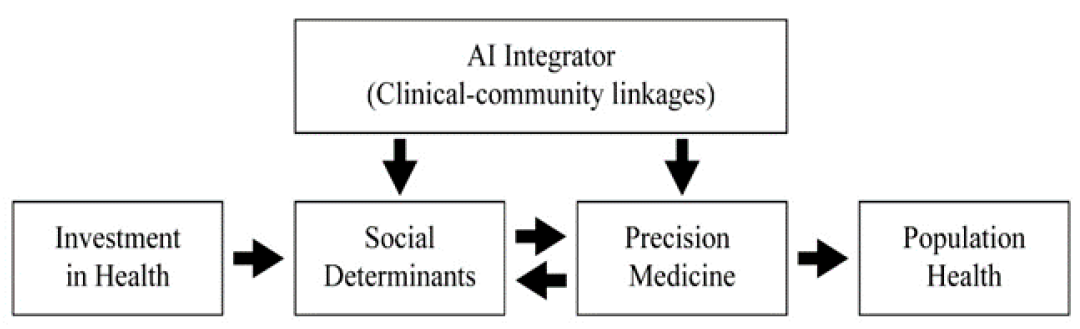

Figure 1. Roadmap to population health.

A nation's health depends on the level of investment in three tracks: information infrastructure (hardware as well as workforce development), healthy communities (SDOH), and medical assets (precision medicine). AI is considered as an integrator to make information on patients and community health characteristics available to healthcare providers. We believe that in order to achieve a maximally healthy community, healthcare providers should and must have access to patient information not only for their personal medical history but also for the community characteristics where they live and interact. Imbalanced investment on any one of these tracks would not achieve population health at its full potential.

\subsection{Investing in Health Technology Infrastructure}

The literature appears to suggest that the investment of technologies in healthcare yields dividends in both cost savings and better health outcomes [24]. However, a rapid deployment of digital communication devices creates a "digital divide" between those who have access to technologies and digital literacy, and those who do not have access to such technologies. In healthcare, the digital divide can lead to disparities in patient portal adoption, telehealth care access, or the ability to utilize patient-facing management software such as online appointment schedulers. Nearly 75\% of households either lack or are unaware of telehealth options, or both [25]. Black patients were four times more likely than white patients to visit the emergency department, not telehealth, during the pandemic's initial surge [26]. Access to digital technologies assumes that every citizen can participate in the healthcare improvement process. Investment in digital infrastructure is a necessary first step toward achieving the goal of minimizing or eliminating the digital divide. One study shows that investment in key clinical health AI applications could create $\$ 150$ million in annual savings for the U.S. healthcare economy by 2026 [24].

\subsection{Investing in Social Determinants of Health}

Data collection in social determinants of health (personal and household income and expenses by items; neighborhood characteristics; education; food security; and community characteristics including safety, social interaction on a personal basis, healthcare accessibility, etc.) should be easier with digital networks. However, this is a challenging task, as it involves and pertains to sensitive personal information. Since the Social Deprivation Index (SDI) requires information on an individual basis (not households or blocks) in very small communities of residence (blocks or streets in neighborhoods), people are reluctant to reveal sensitive information. For instance, information such as criminal record, alcohol 
addiction, numbers of marriages/divorces, child/spouse abuse, places they spend their income, welfare checks, etc. are personal, private, and pervasive. As a result, the information collected is messy, insufficient, or not reflective of the community characteristics. Nevertheless, the information listed above is essential in creating the Social Deprivation Index. There are a few studies that have successfully collected such information in creating the SDI [22].

\subsection{Investing in Precision Medicine}

The United States has invested an enormous amount of resources in medicine, drugs, and personnel workforce in this area, more than any other advanced country. The 2019 statistics show that this country spent $\$ 3649.4$ billion on healthcare. Expenditures were, in part, $\$ 1191.8$ billion on hospitals (32.7\%), $\$ 564.4$ billion on physicians $(15.5 \%)$, $\$ 335$ billion on drugs $(9.2 \%)$, and $\$ 174.4$ billion on investment (4.8\%) [27]. It is interesting to note that the United States spent less than $5 \%$ of its healthcare spending on investment, while almost $60 \%$ of its spending was on hospitals, physicians, and drugs. It appears that there is room to increase the size of investment, including in information infrastructure and training the workforce in IT areas to make healthcare operations leaner, more efficient, and more effective without increasing healthcare spending.

\subsection{Roles of AI as a Clinical-Community Integrator}

The current healthcare practice in this country is that the two important healthenhancing constructs (SDOH and precision medicine) play separately and independently from each other [16]. We speculate that the improvement of population health requires that these two constructs work together to produce maximum health benefits. AI could and should play a role as an integrator/communicator between these two constructs. Since important healthcare information is now in the hands of healthcare providers, the social determinants of a patient should be merged with the patient's health-specific information from their health records. AI by machine learning can generate and map the treatment protocol, and the sum of individual health would then be closer to the nation's overall population health.

\section{Discussion and Limitations}

Although digital transformation in healthcare provides numerous benefits, there are issues and challenges in adopting AI and other technologies. For example, according to the 2018 Price Waterhouse Cooper survey, only 38\% of chief executive officers of U.S. healthcare systems reported a digital component in their overall strategic plan [28]. Over 90\% of respondents in the same survey pointed to data-protection and privacy regulations such as the 1996 Health Insurance Portability and Accountability Act, and the expansion of rules and penalties under the Health Information Technology for Economic and Clinical Health Act as factors limiting digital strategies at a micro level. Addressing the same issues of limiting the use of digital devices, Mehta et al. [1] cite three reasons: (1) reliability and availability of data, (2) matching AI tools to the right provider, and (3) an insufficient focus on ensuring that the workforce is motivated to use these tools effectively.

Crabb [29] broadens the limitations and challenges in using AI in healthcare industries. Many healthcare providers are still operating with technologies and data infrastructures that are incompatible with new, high-speed technologies. In addition, there is a conflict in reimbursement models (fee-for-service vs. value-based care). Despite some studies showing a positive return on investment, the evidence is limited. From a personnel standpoint, there is resistance to change with healthcare systems, especially from physician groups. Finally, existing studies have not addressed the impact of digital health on the overall healthcare ecosystem. The speed of technological innovation has outpaced the industry's willingness and/or capability to adopt new technologies, leaving healthcare leaders further behind the roadmap to achieve their strategic goals. Investment in information infrastructure, including in workforce training, is the best way to achieve improved 
population health. Finally, this study has not addressed privacy issues in the application of AI to social determinants of health. One of the major issues that has hampered the collection of personal and private information is the fear that such information may invade a participant's personal life. This issue must be addressed in a public forum, and some types of legislation may be needed to protect personal information before AI is fully utilized in healthcare fields.

\section{Conclusions}

Despite the many challenges, AI in healthcare industries will continuously play a significant role as we face four important new waves in the healthcare market: consumerism (on-demand health services), healthcare reform (health as a public good), efficiency (cost and process), and the patient-clinician-caregiver relationship (personal interaction with caregivers). We argue that investment in three areas (IT infrastructure, $\mathrm{SDOH}$, and precision medicine) would be the best way to achieve better population health.

Author Contributions: Conceptualization, I.-W.G.K.; investigation, I.-W.G.K. and S.-H.K.; project administration, D.M. writing-review and editing. All authors have read and agreed to the published version of the manuscript.

Funding: This research received no external funding.

Institutional Review Board Statement: This study did not require ethical approval as this study does not involve in human as a research protocol.

Informed Consent Statement: Not applicable for studies not involving humans.

Data Availability Statement: Not applicable.

Conflicts of Interest: The authors declare no conflict of interest.

\section{References}

1. Mehta, M.C.; Katz, I.T.; Jha, A.K. Transform Global Health with AI. NEJM 2020, 382, 791-793. [CrossRef] [PubMed]

2. Frankel, T. Pandemic Led to Fewer Sports Injuries, More Firework and Power-Tool Accidents. Washington Post, 4 March 2021. Available online: https: / /www.washingtonpost.com/business/2021/03/04/pandemic-home-injuries (accessed on 26 March 2020).

3. Kent, J. How Machine Learning is Transforming Clinical Decision Support Tools. Health IT Analytics. Available online: https:// healthitanalytics.com/features/how-machine-learning-is-transforming-clinical-decision-support-tools (accessed on 26 March 2020).

4. Stanfill, M.H.; Marc, D.T. Health Information Management: Implications of Artificial Intelligence on Healthcare Data and Information Management. IMIA Yearb. Med. Inform. 2019, 28, 56-64. [CrossRef] [PubMed]

5. Miriovsky, B.J.; Shulman, L.N.; Abernethy, A.P. Importance of health information technology, electronic health records, and continuously aggregating data to comparative effectiveness research and learning health care. J. Clin. Oncol. 2012, 30, 4243-4248. [CrossRef] [PubMed]

6. Reddy, M. Digital Transformation in Healthcare in 2021: 7 Key Trends. Digital Authority Partners, 4 January 2021.

7. Ganguli, I.; Gordon, W.J.; Lupo, C.; Sands-Lincoln, M.; George, J.; Jackson, G.; Rhee, K.; Bates, D.W. Machine Learning and the Pursuit of High-Value Health Care. NEJM Catal. 2021, 1, 1-14. [CrossRef]

8. Halamka, J.; Cerrato, P. The Digital Reconstruction of Health Care. NEJM Catal. 2021, 1, 1-12. [CrossRef]

9. Fox, S.; Duggan, M. Health Online 2013. Pew Research Center. 15 January 2013. Available online: https://www.pewresearch.org/ internet/2013/01/15/health-online-2013 (accessed on 26 March 2020).

10. World Health Organization. Global Strategy on Digital Health 2020-2025; WHO: Geneva, Switzerland, 2015.

11. Kraus, S.; Schiavone, F.; Pluzhnikova, A.; Invernizzi, C.A. Digital transformation in healthcare: Analyzing the current state-ofresearch. J. Bus. Res. 2021, 123, 558-567. [CrossRef]

12. Walsh, C.G.; Johnson, K.B.; Ripperger, M.; Sperry, S.; Harris, J.; Clark, N.; Fielstein, E.; Novak, L.; Robinson, K.; Stead, W. Prospective Validation of an Electronic Health Record-Based, Real-Time Suicide Risk Model Prospective Validation of an Electronic Health Record-Based, Real-Time Suicide Risk Model. JAMA Network Open 2021, 4, e211428. [CrossRef]

13. Zhou, X.; Qui, S.; Joshi, P.S.; Xue, C.; Killiany, R.J.; Mian, A.Z.; Chin, S.P.; Au, R.; Kolachalama, V.B. Enhancing magnetic resonance imaging-driven Alzheimer's disease classification performance using generative adversarial learning. Alzheimer's Res. Ther. 2021, 13, 1-11. [CrossRef] [PubMed]

14. World Health Organization. Social Determinants of Health. 25 September 2017. Available online: www.who.int/social_ determinants/sdh_definition/en/ (accessed on 26 March 2020).

15. Batheja, C.; Meeting People Where They Really Are. Conference Session at Mayo Clinic Transform 2018, 26 September 2018. Available online: www.youtube.com/watch?v=FUPjbWfl0zM (accessed on 26 March 2020). 
16. Kim, S.H.; Kwon, I.W.G. Population health from Social Determinants of Health Prospects: A Global Comparison. J. Hosp. Health Care Adm. 2020, 4, 1-9. [CrossRef]

17. Centers for Medicare \& Medicaid Services. Health community Model Health Community Model; CMS: Baltimore, MD, USA, 2015.

18. Eisenson, H.; Mohta, N.S. Health Care Organizations Can and Must Incorporate Social Determinants. NEJM Catal. $2020,1,1-18$. [CrossRef]

19. Elena, D.; Butler, A.B.; Morgan, A.U.; Kangovl, S. Screening for Unmet Social Needs: Patient Engagement or Alienation? NEJM Catal. 2020. [CrossRef]

20. Fraze, T.K.; Brewster, A.L.; Lewis, V.A.; Beidler, L.B.; Murray, G.F.; Colla, C.H. Prevalence of screening for food insecurity, housing instability, utility needs, transportation needs, and interpersonal violence by US physician practices and hospitals. JAMA Network Open 2019, 2, e1911514. [CrossRef] [PubMed]

21. Butler, D.C.; Petterson, S.; Phillips, R.L.; Bazemore, A.W. Measures of Social Deprivation That Predict Health Care Access and Need within a Rational Area of Primary Care Service Delivery. Health Serv. Res. 2013, 48, 539-559. [CrossRef]

22. Maroko, A.R.; Doan, T.M.; Armo, P.S.; Hubel, M.; Yi, S.; Viola, D. Integrating Social Determinants of Health with Treatment and Prevention: A New Tool to Assess Local Area Deprivation. Preventing Chronic Dis. 2016, 13. [CrossRef] [PubMed]

23. Snyder, H. Literature review as a research methodology: An overview and guidelines. J. Bus. Res. 2019, 104, 333-339. [CrossRef]

24. Collier, M.; Fu, R.; Yin, L.; Christiansen, P. Artificial Intelligence (AI): Healthcare's New Nervous System. Accenture. 2017. Available online: https://www.accenture.com/_acnmedia/PDF-49/Accenture-Health-Artificial-Intelligence.pdf (accessed on 26 March 2020).

25. J.D. Power. U.S. Telehealth Satisfaction Study. 2019. Available online: https://www.jdpower.com/business/press-releases/2019 -us-telehealth-satisfaction-study (accessed on 28 October 2019).

26. Weber, E.; Miller, S.J.; Astha, V.; Janevic, T.; Benn, E. Characteristics of telehealth users in NYC for COVID-related care during the coronavirus pandemic. J. Am. Med. Inform. Assoc. 2020, 27, 1949-1954. [CrossRef] [PubMed]

27. American Medical Society. Trends in Health Care Spending. 2019. Available online: https://www.ama-assn.org/about/research/ trends-health-care-spending (accessed on 26 March 2020).

28. Yednak, C. Will digital show an ROI for Healthcare? PWC Health Research Institute, 18 February 2020.

29. Crabb, S. Digital Health: Promise, Perils and Perspective. J. AHIMA 90 2019, 5, 32-35. 